\title{
Adaptation, science de la durabilité et pensée planificatrice
}

\author{
Vincent Berdoulay ${ }^{1}$, Olivier Soubeyran ${ }^{2}$ \\ ${ }^{1}$ Géographe et aménagiste, Université de Pau et des Pays de l'Adour, CNRS, UMR 5603, Laboratoire SET, 64000 Pau, France \\ ${ }^{2}$ Géographe et aménagiste, Université Joseph-Fourier, CNRS, UMR 5194, Laboratoire PACTE, 38040 Grenoble, France
}

L'injonction à l'adaptation au changement climatique officialisée en 2008 par le Grenelle de l'environnement pose des problèmes pratiques et théoriques considérables, en raison des incertitudes très fortes qui portent à la fois sur la nature, l'ampleur et la temporalité de ce changement. C'est en particulier le cas dans le domaine de l'aménagement où les approches traditionnelles de la planification paraissent inapplicables. La grande originalité de cet article est de proposer de dépasser cette contradiction en articulant les questions d'adaptation et de planification avec les principes qui sont ceux des «sciences de la soutenabilité ». S'appuyant sur celles-ci, dont l'essor remonte aux trente dernières années, les deux auteurs proposent de ne pas opposer aux démarches "d'anticipation planificatrice» (top down), une logique bottom up privilégiant les capacités d'adaptation et de résilience de la société civile, mais plutôt d'articuler ces deux types d'action dans une approche « d'adaptation transformatrice », partie intégrante de stratégies territoriales de développement durable.

La Rédaction

Mots-clés :

environnement, changement climatique, adaptation, théorie de la planification, science de la durabilité

\section{Keywords:} environment; climate change; adaptation; planning theory; sustainability science

\begin{abstract}
Résumé - L'article met en relation la question de l'adaptation au changement climatique avec celles de la durabilité et de l'action planificatrice. Le champ de préoccupations que dénote la science de la durabilité sert à recadrer l'adaptation au sein d'une approche planificatrice renouvelée. Après avoir montré la portée à la fois scientifique et aménagiste d'une science de la durabilité telle que certains la dessinent actuellement, l'article se concentre sur l'enjeu crucial de la résilience, avant d'en déduire l'intérêt d'une adaptation transformatrice en aménagement.
\end{abstract}

\begin{abstract}
Adaptation, sustainability science, and planning theory. This article sets the issue of adaptation to climate change in relationship to that of sustainability and action in planning theory. The broad field of concern, which sustainability science designates, helps in providing a new framework to deal with adaptation in a novel planning approach. The article first gives an overview of what this scientific field is about, through a brief history of the emergence of sustainability science. This makes it possible to then cast a new light on the issue of adaptation to climate change; consequently, vulnerability and resilience come up as important foci for planning. Finally, the whole conception of planning is shown to be concerned: a transformative approach to adaptation is suggested, showing how difficulties in French territorial planning could be bypassed by looking at adaptation through the lenses of sustainability science.
\end{abstract}

La préoccupation pour le changement climatique et pour l'adaptation qui doit s'ensuivre est venue récemment amplifier l'enjeu environnemental et plus particulièrement celui de la durabilité de l'action humaine sur la Terre ${ }^{1}$. C'est ainsi que l'adaptation au changement climatique a fait irruption dans les débats concernant les moyens à mettre en œuvre pour satisfaire un objectif de durabilité (Magnan, 2010). La question qui se pose est de savoir dans quelle mesure l'idée d'adaptation peut modifier la pensée planificatrice en matière $\mathrm{d}$ 'aménagement. Ce qui intéresse alors est moins une adaptation qui serait simplement réactive qu'une façon de l'envisager comme composante d'une planification permettant l'anticipation et l'élaboration de stratégies face à un nouveau contexte, celui du changement climatique.

Auteur correspondant : V. Berdoulay, vincent.berdoulay@univ-pau.fr

1 Cet article s'inscrit dans le cadre d'une recherche financée par le programme CDE (Concertation Développement Environnement) du ministère français de l'Écologie, du Développement durable, des Transports et du Logement. 
Par ailleurs, on sait que le développement durable fait l'objet d'un large consensus que les politiques publiques s'efforcent de reprendre à leur compte. Elles ont induit la recherche d'outils scientifiques, conceptuels ou techniques, pour avancer vers cet objectif. Parmi ceux-ci se distingue un courant particulier de mise en cohérence de tout ce champ de réflexions et d'expériences depuis le tout début de ce siècle, à savoir l'idée d'une « science de la durabilité ». Elle vise à s'appuyer sur les apports de la recherche scientifique et technologique afin de trouver les moyens d'atteindre les objectifs du développement durable. Mais sa portée en aménagement, peu traitée jusqu'à présent, mérite d'être développée. C'est ce à quoi nous nous attacherons dans cet article. Il va de soi que notre propos n'est pas de plaquer une conception particulière - la science de la durabilité - telle qu'elle émerge actuellement en certains lieux du savoir et de l'action, mais plutôt de voir ce que ce faisceau de préoccupations apporte d'original pour nous aider à repenser l'aménagement à l'aune de la durabilité.

Notre point de vue est que l'adaptation au changement climatique doit être mise en relation avec celles de la durabilité et des modalités de l'action planificatrice. Ce sont les termes et conséquences de cette mise en relation qui retiendront ici notre attention. Plus particulièrement, nous chercherons à montrer l'intérêt d'une articulation entre ces trois types de préoccupation. Comment l'adaptation, abordée par le biais de la science de la durabilité, interpelle-t-elle la pensée planificatrice ? Comment celleci pourrait-elle se saisir de l'adaptation pour avancer? Comment la concertation peut-elle être utilement convoquée? $\mathrm{Au}$ fond, comment penser l'adaptation sans retomber dans les ornières dont la planification a du mal à sortir ? Depuis les années 1970, la question environnementale incarnait en effet la montée en puissance des situations d'incertitude, de surprise, face auxquelles le paradigme de la planification rationnelle, toujours prégnant, se trouvait particulièrement mal à l'aise pour répondre (Alexander, 1984 ; Soubeyran, 2000 ; Chalas et Soubeyran, 2010 ; Gross, 2010). Or, le changement climatique a rendu encore plus incontournables et structurantes ces situations pour la planification (Kay, 2010 ; Hulme, 2009 ; Giddens, 2011 ; Zaccaï et al., 2012).

Pour répondre à ce questionnement, il faut d'abord veiller à dissiper quelques malentendus à propos de la science de la durabilité. Par l'intermédiaire d'un bref retour sur l'histoire, nous allons rappeler ce qu'elle n'est pas et ce qu'elle cherche à être. Il sera alors possible de montrer qu'il existe d'importants enjeux dans la façon de concevoir l'adaptation en rapport avec la pensée planificatrice. Enfin, nous suggérerons, à l'aide de l'exemple français, comment l'adaptation, cadrée par la science de la durabilité, pourrait aider à penser autrement la planification.

\section{La science de la durabilité : une invitation à franchir le Rubicon}

Sur fond de recherches internationales sur les rapports entre science et développement durable est née l'idée d'une science de la durabilité visant leur mise en cohérence. De nombreux travaux abordaient déjà certains de ces rapports sous des angles différents, analysant notamment leurs dimensions gestionnaires, écologiques ou épistémologiques (Holling, 1978; Clark et Munn, 1986). Les réflexions théoriques faisaient suite à des expériences déjà anciennes en matière d'aménagement (Berdoulay et Soubeyran, 1996, 2000), mais elles se sont tout particulièrement développées au croisement de la question des risques et de la vulnérabilité (Burton et al., 1978 ; Sachs, 1980 ; Beck, 2001 [1986] ; Fabiani et Theys, 1987). Même si celles-ci n'ont pas été sans influence, 1 'effort inédit de mise en cohérence que défend la science de la durabilité offre l'opportunité d'une rupture conceptuelle.

Cette expression «science de la durabilité » est née aux États-Unis au cours des travaux (1995-1999) de la commission sur le développement durable créée par le National Research Council en coopération avec l'Académie nationale des sciences, afin de réfléchir aux moyens de promouvoir la durabilité. L'expression « science de la durabilité » est venue spontanément de la bouche du président de l'académie (Bruce Albert) pour désigner les travaux de ce groupe de 25 scientifiques présidé par un physicien (Shere Abbott), avec pour vice-présidents un géographe (Robert Kates) et un écologue de formation (William Clark). Il s'est en effet révélé pratique de donner un nom au faisceau des recherches scientifiques qui, internationalement, œuvraient en faveur du développement durable. En essayant de préciser les enjeux scientifiques et technologiques d'une transition qui mènerait à cet objectif, la commission a ainsi contribué à orienter la façon d'approcher scientifiquement la question de la durabilité (NRC, 1999; Kates, 2000). Un nombre croissant de travaux et d'institutions de recherche et d'enseignement se référant à l'appellation «science de la durabilité » établit un relatif consensus autour de certains objectifs, principes et méthodes (Kates et al., 2001; Komiyama et Takeuchi, 2006 ; Clark et Dickson, 2003 ; Clark, 2007). Ce que nous allons ici souligner, c'est ce que cette orientation apporte comme rupture par rapport à des pratiques planificatrices établies : face à celles-ci, elle invite à franchir le Rubicon.

De façon générale, l'utilisation du terme de science ne renvoie pas à l'idée d'une discipline fortement structurée et autonome, reposant sur un socle de méthodes applicables partout, même s'il existe quelques velléités en ce sens. Il s'agit plutôt d'un champ de préoccupations scientifiques et technologiques, qui se définit principalement 
par les problèmes liés à la durabilité. En revanche, ce champ se veut essentiellement transdisciplinaire, afin de saisir la complexité des relations homme/environnement en dépassant les barrières qui existent entre les disciplines traditionnelles. Ce souci d'intégrer ce qui est étudié séparément se manifeste aussi à propos des échelles de temps et d'espace trop souvent réifiées. Il caractérise aussi la volonté, tout à fait centrale, de produire des connaissances utilisables pour promouvoir la durabilité.

En effet, 1'idée est de ne pas distinguer entre savoir, savoir-faire, savoir-agir, ou encore entre science fondamentale et science appliquée. La science de la durabilité vise à produire des résultats que l'on peut mettre en pratique. La complexité des dynamiques qui relient les systèmes humains et environnementaux rend peu profitable la distinction entre connaissance et action. C'est qu'il est souvent impossible de conforter l'une sans l'aide de l'autre. Ce point de vue, typiquement pragmatiste, insiste sur le fait que la connaissance s'établit dans l'action, c'est-à-dire que l'on apprend tout en agissant. Ni purement théorique, ni seulement appliquée, la science de la durabilité promeut une recherche fondamentale à visée pratique, comme ont pu le réaliser des scientifiques tels que Pasteur (Stokes, 1997 ; NRC, 1999).

Il s'agit donc pleinement d'une science de l'action orientée vers la durabilité. En cela, cette science reconnaît qu'elle repose sur des valeurs. Il s'agit autant de celles des scientifiques que de celles de la société en général, qui toutes valorisent la solidarité et la sensibilité à l'écologie. Comment décider ce qui doit être considéré comme durable et comment procéder ? Entre l'anthropocentrisme et l'écocentrisme, entre l'esthétique et l'économisme, il existe toute une gamme de valeurs placées sur ce dont l'homme comme la nature ont besoin. En conséquence, toute une partie de l'effort de la science de la durabilité porte sur les liens entre valeurs, attitudes et comportements effectifs qui conditionnent l'action en faveur de la durabilité (Leiserowitz et al., 2006). Cela ouvre d'intéressantes perspectives sur la place de l'enjeu éthique au sein du processus de planification. En effet, le discours éthique, en désignant ce qui est désirable et ce qui ne l'est pas, ceux qui sont responsables et ceux qui ne le sont pas, les bénéficiaires et les éventuelles victimes, conduit à réduire les incertitudes d'un projet. C'est pourquoi l'aménagement au nom de l'adaptation, s'il veut échapper aux ornières de la planification dite rationnelle où certaines valeurs sont plaquées sur l'espace du projet, doit intégrer au sein de son processus même une préoccupation éthique. Celle-ci, parce qu'elle se travaille avec la population concernée, redonne tout son sens à la concertation, au-delà de la simple consultation. Ce sont donc bien des pratiques réflexives auxquelles peut conduire l'adaptation en aménagement (Berdoulay et Soubeyran, 2012).
Ce faisant, et par souci de l'action, cette approche scientifique de la durabilité reconnaît, à côté d'un système naturel, l'autonomie d'un système humain. Comme la durabilité cherche à rendre compatible le bien-être des humains et la capacité de la nature à faire perdurer la vie, il s'agit d'approcher ces deux grands systèmes comme étroitement couplés, c'est-à-dire d'étudier la complexité de leurs interactions (Liu et al., 2007). Il s'agit là d'un défi méthodologique mais aussi épistémologique récurrent, qui demeure central dans la dynamique de la recherche qui n'est pas seulement interdisciplinaire, comme le cas de la géographie l'a toujours bien illustré (Glacken, 20002007 ; Claval, 2001).

Ce qui est intéressant dans toutes ces démarches, c'est que, du point de vue de l'action, l'incertitude demeure une préoccupation centrale. Tant en ce qui concerne les systèmes humains que naturels, et $a$ fortiori leurs interactions, il n'est pas possible de prévoir le cours des choses et encore moins de le façonner à l'avance. En ce sens, ces systèmes et leurs interactions sont vus comme contingents. Non seulement les analyses et les modèles mobilisés doivent en tenir compte, mais c'est aussi la façon de penser l'action qui doit être complètement revue par rapport à une anticipation et une prévision traditionnelles qui visaient précisément à éliminer cette incertitude. Trois aspects peuvent être soulignés en rapport avec la durabilité.

Tout d'abord, ce qui échappe à un cadrage scientifique classique, c'est-à-dire cette incertitude présente dans la nature et les comportements humains, doit être intégré au cœur de l'action planificatrice. C'est là qu'une certaine conception de la concertation peut jouer un rôle décisif. Il ne s'agit pas tant de faire participer des catégories nombreuses de la société à la construction d'un projet - même si ce souci est légitime et peut s'avérer utile. L'originalité du point de vue de la science de la durabilité est de voir dans la concertation un des moteurs de l'action. L'idée est de s'ouvrir à tous les acteurs possibles, car il est difficile d'estimer à l'avance ceux qui pourraient se révéler les plus performants selon les contingences du futur. Ainsi, la science de la durabilité est intrinsèquement démocratique ; elle ne pose pas des catégories prédéterminées de population qui seraient pertinentes ; elle convie au contraire à reconnaître le potentiel de toute forme d'organisation sociale. Le scientifique, l'élu et tout autre habitant sont potentiellement coauteurs des solutions à mettre en œuvre en faveur de la durabilité. Ils sont importants à toutes les étapes du processus, de la création de connaissances à leurs diffusion et utilisation (Lynam et al., 2007).

Ensuite, l'attention portée à l'incertitude et la volonté d'en tirer parti se retrouvent dans la façon de penser les espaces d'intervention. Alors que le texte fondateur des politiques de développement durable - le rapport 
Bruntland (CMED, 1987) - procédait de façon sectorielle, la science de la durabilité a vite pris conscience de la diversité spatiale des interactions entre systèmes humains et naturels à la surface de la Terre, au point qu'elle doit être vue comme toute entière «place-based», c'est-à-dire comme reposant sur la prise en compte des lieux (NRC, 1999). Cette façon d'envisager l'analyse et l'action en matière de durabilité ne doit toutefois pas être axée sur des territoires prédéfinis, avec les limites spatiales et les pouvoirs institués qui leur sont associés. Au contraire, le recours à l'idée de lieu renvoie à la volonté de mobiliser librement des échelles différentes pour saisir ce que les contingences peuvent faire survenir comme nouveaux paramètres à intégrer. Cela permet aussi de voir comment ces échelles d'analyse et d'action se combinent afin de relier les préoccupations pour la planète avec celles pour le local.

Enfin, l'importance à accorder à l'incertitude incite à adopter une approche de l'action qui reste constamment attentive à la fluidité des événements, aux retournements possibles, aux expériences et aux apprentissages. En somme, faute de pouvoir tracer à l'avance une voie précise pour agir, la science de la durabilité ne se borne pas à accepter cette contrainte, elle la transforme pour structurer sa démarche. En effet, par la métaphore du voyage (NRC, 1999), elle préconise un apprentissage dans l'action qui passe par une adaptabilité constante aux contingences et aux résultats des expériences. C'est pourquoi elle mobilise les outils de la gestion adaptative (Holling, 1978; Folke et al., 2005). Les systèmes ne se comportent pas toujours de façon linéaire et ne sont pas prédictibles en fonction de leur état d'origine; ils sont adaptatifs, de sorte que des propriétés émergentes vont conditionner de façon nouvelle la durabilité. Ce qui importe ici, c'est de concevoir la durabilité plus comme un processus à favoriser actuellement que comme un objectif pour le futur.

Ainsi, la question de l'adaptation qui est posée de nos jours, suite à la prise de conscience des effets du changement climatique, entre en résonance avec la démarche de la science de la durabilité. Or, elles ont été toutes deux traversées par la préoccupation de faire face aux risques environnementaux. Les recherches sur les aléas et la vulnérabilité ont conditionné la réflexion sur l'adaptation, qui s'est tout particulièrement portée sur la résilience.

\section{L’adaptation et la résilience}

Les recherches sur la vulnérabilité aux catastrophes naturelles ou technologiques ont servi de creuset pour réfléchir aux capacités de récupération des populations impactées. Il est révélateur que les travaux pionniers sur les risques naturels aient opté - après quelques hésitations - pour le terme $\mathrm{d}^{\prime}$ « ajustement » plutôt que pour celui d' « adaptation » pour désigner ce qui était fait ou devait être fait pour pallier les catastrophes (Burton et al., 1978). Le schéma initial de réflexion sur les risques semblait donc adopter une perspective où les mesures préconisées ne visaient pas à changer la structure fondamentale des systèmes en place, comme le terme d'adaptation aurait pu le faire croire. En fait, et paradoxalement, la perspective des auteurs n'en était pas si éloignée : ils argumentaient en faveur d'une transformation - voire une rupture - dans la façon de gérer l'interface des systèmes naturels et humains, qui, autrement, allaient conduire aux mêmes catastrophes (par exemple, plus de travaux hydrauliques peut conduire à plus de dégâts d'inondation). L'élargissement des réflexions apporté par la science de la durabilité a confirmé cette perspective transformationnelle (Kates et al., 2012). De plus, le fait de raisonner en planification sur des possibilités de catastrophes a réinstallé au cœur de la pensée planificatrice l'importance de l'incertitude. On comprend alors que la résilience ait fait l'objet d'une attention particulière.

En matière de théorie de la planification, il est clair que l'adaptation dépasse la logique de l'« incrémentalisme » (Lindblom, 1959, 1979; Soubeyran, 1988). La science de la durabilité, qui donne sens et portée à l'adaptation, ambitionne d'incarner un nouveau paradigme, voire " une seconde révolution copernicienne », loin de la simple recherche d'ajustements mineurs : l'adaptation s'inscrit comme l'une des modalités d'un «nouveau contrat entre science et société » (Clark et al., 2004, p. 23), irréductible à la seule fonction d'ajustement. À ce titre, l'adaptation ne peut que rompre avec une vision incrémentale de la planification.

Mais ce qui frappe aussi du point de vue de la science de la durabilité, c'est que l'adaptation « incrémentale » (à base de simples ajustements) peut certes réduire la vulnérabilité pour des événements fréquents, c'est-à-dire de faible ampleur ; mais elle produit presque inévitablement aussi un accroissement de la vulnérabilité pour des événements extrêmes. La catastrophe provoquée en 2005 par l'ouragan Katrina à La Nouvelle-Orléans en a malheureusement été la démonstration éclatante (Colten et al., 2008). Rehausser un peu les digues à la suite de chaque inondation n'a pas permis de contenir un phénomène extrême et en a aggravé les conséquences, avec l'endiguement des eaux à l'intérieur de la ville. La possible survenue d'un tel événement exige de repenser autrement l'exposition au risque de la ville.

L'adaptation devient le moment où l'on franchit le Rubicon, c'est-à-dire où l'on s'adapte en transformant radicalement la vision moderne des relations société/ nature et les actions qui en découlent. Cela ne veut pas dire que les ajustements sont bannis du vocabulaire de la science de la durabilité. Mais ils interviennent une fois la transition paradigmatique entamée. 
Toujours du point de vue de la théorie de la planification, on pourrait croire que ce rejet de l'incrémentalisme, ajouté à l'ambition de la science de la durabilité de fonder un nouveau paradigme, conduit à un retour à la « planification rationnelle », ce comprehensive planning si critiqué pendant les années 1970 (Soubeyran, 1988). S'agirait-il d'un nouveau comprehensive environnemental planning issu des années 1970 (Kates, 1974) mais rafraîchi par la rhétorique du développement durable? En fait, il n'en est rien. Ce sont plutôt les leçons de l'échec de la planification rationnelle qu'ont intégrées les partisans d'une science de la durabilité : pas de visée totalisante (comprehensive), et méfiance vis-à-vis d'une approche par le haut (top-down). C'est que la volonté forte de maîtriser le changement ou de le canaliser par des systèmes de contrôle rigides s'est avérée trop souvent contreproductive. On se méfie donc des politiques censées provoquer les adaptations attendues mais qui, de fait, fragilisent les capacités adaptatives des populations soumises à ces injonctions adaptatives (Folke et al., 2002a).

Autrement dit, du point de vue de la planification, il semblerait que le lieu essentiel où se joue l'adaptation serait celui de la population locale. Un tel déplacement de perspective s'appuie le plus souvent sur des exemples de catastrophes, à la fois naturelles et sociales, telle Katrina. Par définition, une catastrophe signifie effondrement du sens, de tout ce qui était programmé (Entrikin, 2007). Elle constitue donc bien la preuve de l'inadaptation des réponses envisagées pour la contrer. Or, on a pu constater que bien souvent ce sont les acteurs non institutionnels, des "répondeurs de l'ombre » (Colten et al., 2008, p. 43), qui ont su trouver des réponses à des situations d'urgence imprévues. Ainsi, l'enjeu de l'adaptation devient celui du renforcement de la capacité adaptative $\mathrm{du}$ niveau local, autrement dit celui de sa résilience (Wilbanks et Kates, 2010). C'est donc la résilience qui devient la notion-clé d'une science de l'action. Celle-ci est tendue non plus vers le contrôle du changement dans des systèmes supposés stables, mais vers l'amélioration de la capacité des systèmes sociaux et écologiques à faire face, à s'adapter et à façonner le changement dans un monde où le futur est imprédictible et la surprise probable (Folke et al., 2002a ; Turner et al., 2003).

La résilience constitue ainsi autant un enjeu qu'une notion centrale pour penser l'adaptation dans un contexte de durabilité. La résilience peut se définir comme la capacité d'un système complexe social et écologique à absorber des chocs tout en maintenant ses fonctions essentielles, voire en augmentant ses capacités auto-organisatrices, d'apprentissage et d'adaptation. Ainsi, adaptation et capacité adaptative sont enchâssées dans la notion de résilience, qui devient l'antonyme de la vulnérabilité (Folke et al., 2002b). En termes de planification, la résilience se définit comme la capacité d'une collectivité à se préparer (anticiper), à répondre (pendant l'urgence de la catastrophe), à pouvoir récupérer une fois la catastrophe passée, et enfin à pouvoir tirer les leçons pour diminuer sa vulnérabilité.

Du fait même que de telles perspectives d'action ont été renforcées dans leur légitimité par la survenue de catastrophes telles que celle de Katrina, on a pu être tenté d'opposer l'adaptation à la capacité adaptative. La première s'incarne dans les politiques et les programmes construisant les cadres de l'anticipation et imposant par le haut des conduites jugées adaptées. Mais puisqu'il y a eu catastrophe et crise, c'est que l'adaptation pensée et conduite par le haut a échoué. En revanche, à l'autre bout de la chaîne des événements, il y a la population locale qui subit les conséquences de la catastrophe, mais aussi d'une planification contreproductive. Celle-ci n'a pas permis d'éviter la catastrophe, et en plus elle a souvent neutralisé la capacité adaptative de la collectivité (Folke et al., 2002b, p. 8). Or, le salut de cette population peut venir de sa résilience et en particulier de sa capacité adaptative. C'est pourquoi on a pu penser qu'elle seule était capable de faire face à la surprise, à l'inattendu, bref à l'effondrement du sens qui caractérise la survenue d'une catastrophe.

Mais les études qui ont été produites sur la résilience " communautaire », notamment pour tirer les leçons de Katrina, sont loin d'être aussi binaires (Colten et al., 2008 ; Wilbanks et Kates, 2010). On y discerne une relative imbrication de ce qui relève de l'intervention par le haut avec ce qui procède de l'initiative populaire, comme si l'une était une condition d'amélioration de l'autre et réciproquement. Cela vient faire écho à la démarche valorisée par la science de la durabilité : il s'agit d'approcher l'adaptation non comme simplement planifiée par le haut (une adaptation passive) ni comme reposant entièrement sur l'initiative locale, mais plutôt comme une résultante de leur interaction. Étant donné qu'une telle voie ne se dessine qu'au prix d'une transformation des systèmes impliqués, il faut ainsi envisager une conception transformatrice de l'adaptation.

\section{Pour une adaptation transformatrice}

Afin de mieux comprendre la portée de ce qu'une conception transformatrice de l'adaptation implique pour la pensée planificatrice, il est intéressant de la mettre en contrepoint de ce qui s'est récemment passé en France, pays où la tradition de planification territoriale est fortement ancrée. C'est pourquoi nous ferons d'abord référence ici, quoique très schématiquement, aux liens qui existent entre planification, durabilité et adaptation dans le contexte français.

C'est en étroite association avec la question du changement climatique que l'adaptation est entrée il y a une 
dizaine d'années dans le vocabulaire de la planification française, impulsée notamment par les différents rapports que l'Observatoire national sur les effets du réchauffement climatique a produits depuis sa création en 2001 (Onerc, 2005, 2007, 2010, 2012), et grâce aussi aux travaux de l'Institut français de l'environnement (Ifen) sur les indicateurs du développement durable (Lavoux et Theys, 2001). Mais il n'est pas faux de rappeler que l'injonction de l'adaptation en aménagement est venue massivement du processus de concertation dit du Grenelle de l'environnement (2007), tant du point de vue académique que professionnel. Certes, des expériences établissant des plans climat, notamment pour de grandes agglomérations, ont vu le jour auparavant, mais elles ont privilégié l'atténuation (Onerc, 2010). Toutefois, l'injonction à penser en termes d'adaptation faite par le Grenelle de l'environnement ne plaçait pas celle-ci au centre de la démarche planificatrice, l'atténuation paraissant plus compatible avec la reformulation des politiques publiques.

La stratégie d'adaptation a ainsi été considérée comme complémentaire de celle de l'atténuation pour diminuer les impacts du changement climatique, et ce, aux différentes échelles territoriales (des plans climats territoriaux au plan national d'adaptation). La démarche planificatrice a ainsi eu tendance à suivre une logique qui consiste à diagnostiquer les territoires du point de vue de leur vulnérabilité et à chercher des réponses dans leur adaptation.

La problématique de l'adaptation a donc été principalement arrimée en France à celle de la vulnérabilité. Cela pose deux types de problèmes. D'une part, on sait que la vulnérabilité peut recouvrir des acceptions différentes, auxquelles sont alors associées des définitions différentes de l'adaptation et donc des solutions différentes (Burton et al., 2002 ; Füssel, 2007), sans compter qu'il n'est pas évident de répondre à la question : entre vulnérabilité et capacité d'adaptation, qu'est-ce qui vient en premier (O'Brien et al., 2004) ? D'autre part, nous avons vu que la vulnérabilité est l'antonyme de la résilience : celleci embrasse une logique de compréhension des phénomènes socioécologiques qui dépasse le seul couplage adaptation/vulnérabilité.

En somme, dans le cas français, l'adaptation est fondamentalement enchâssée dans la problématique du changement climatique, lui-même constituant un des points structurants de la réflexion sur l'aménagement et le développement durable. Il est clair qu'on est alors tenté de saisir l'adaptation comme un des rares enjeux qui viennent légitimer un retour de la planification par le haut. On peut même se demander s'il s'agit de planifier l'adaptation d'un territoire ou plutôt de considérer l'adaptation comme une stratégie de planification dans un environnement incertain.
De plus, tout en étant considérées comme une réponse à une véritable rupture (le changement climatique), les politiques et actions menées en France au nom de l'adaptation suivent une logique plutôt incrémentale. Au moins trois raisons concourent à donner à la planification cette orientation. La première est que l'adaptation est conçue comme une stratégie en contexte d'incertitude. Sont alors privilégiées des «stratégies sans regret », tout en évitant qu'elles puissent conduire à des "mal-adaptations » (Onerc, 2010, p. 83). Dans le présent contexte, la notion de «mal-adaptation» se rapproche de celle d'effet pervers, et signifie une augmentation de la vulnérabilité du territoire concerné. Par stratégie sans regret, on entend en général des stratégies élaborées sous contrainte de scénario du pire, mais en supposant qu'il ne se réalise pas. Tout cela mène à une politique de prudence, de petits pas, où l'horizon de l'évaluation reste plutôt le court terme. La seconde raison tient à la très grande réserve des politiques et des élus face à la question de l'adaptation et aux politiques territoriales censées être élaborées en son nom. Enfin, la troisième raison que nous pouvons avancer est que l'adaptation n'est pas associée à un fort mouvement pour un renouvellement de la démarche scientifique autour de la durabilité. C'est bien ce qui avait déjà été remarqué à propos de l'approche du développement durable (Jollivet, 2001). Il est vrai que les ouvrages de référence ayant une portée réflexive et programmatique sur le développement durable n'ont pas privilégié cette perspective épistémologique (Zaccaï, 2002 ; Theys et al., 2010). Une science de la durabilité $n^{\prime}$ a pas vraiment cours en France, en tout cas, pas avec la même légitimité institutionnelle et scientifique qu'aux États-Unis.

On voit donc que, à la lumière des préoccupations de la science de la durabilité, l'adaptation peut être conçue et approchée d'une façon très différente de celle qui est actuellement présente dans la planification française. On peut ainsi mettre en exergue certains défis que la pensée planificatrice doit relever pour mieux se saisir de la question de l'adaptation.

Tout d'abord, l'appel à l'adaptation ne doit pas être uniquement entendu comme une réponse territoriale aux effets du changement climatique. Celui-ci n'est qu'un enjeu parmi d'autres, comme le souligne la science de la durabilité, et il n'est pas celui à partir duquel tout le reste doit s'organiser. Les dynamiques d'intégration entre les sociétés et leur nature sont moins univariées et rendent plus complexes et potentiellement contradictoires les perspectives de compréhension et d'action par l'adaptation seule.

L'adaptation n'a pas à être présentée comme une contrainte, mais plutôt comme une opportunité d'action, un des leviers par lesquels du développement peut être mis en branle. Mais elle l'est uniquement dans la mesure où elle est fortement intégrée parmi d'autres objectifs. 
C'est l'idée de « cobénéfices » bien perçue dans le raisonnement économique (Perthuis et al., 2010). Relevant du temps long, le coût financier ou social de l'adaptation devient acceptable dans la mesure où les bénéfices que l'on peut en tirer sont associés à d'autres qui se situent davantage dans le court terme. Ainsi, des cobénéfices (écologiques, sociaux, économiques, etc.) peuvent apparaître à toute échelle de temps et d'espace (Wilbanks et Kates, 2010). L'adhésion à un processus d'adaptation passe par une perception des gains que vont apporter les nécessaires transformations des relations entre systèmes sociaux et naturels.

Il faut noter que l'attention portée au court terme n'est pas la même que celle qui prévaut dans les stratégies sans regret, pour lesquelles on admet «qu'elles généreront des bénéfices sociaux et économiques indépendamment du changement climatique» (Banque mondiale, 2011, p. 5). Or, ici, c'est au contraire la dépendance des horizons temporels qui fait problème. Il s'agit d'admettre qu'on est confronté à des situations où le court terme est souvent surévalué par rapport aux bénéfices incertains d'une situation future, c'est-à-dire des situations où nous sommes souvent dans l'incapacité de sacrifier l'intérêt immédiat au futur souhaité mais incertain. Et là, les horizons temporels deviennent incompatibles. Pour certains théoriciens de la planification, $c^{\prime}$ est un des aspects du changement climatique qui contribue à en faire un wicked problem ${ }^{2}$, et même un super wicked problem (Levin et al., 2010). On doit donc agir en anticipant sur cette situation - « être faible et le savoir » - rejoignant ainsi les idées d'Elster sur la rationalité imparfaite (Elster, 1987, p. 101).

Comme déjà évoqué ci-dessus, le processus d'adaptation est un processus fondamentalement participatif. Il repose en effet sur tous les acteurs, publics ou privés, quelles que soient leurs fonctions dans la société. Le contexte d'incertitude des actions à prendre dans le futur fait que tout individu ou groupe est potentiellement partie d'une solution à trouver le moment venu. C'est pourquoi la participation doit s'entendre de façon large, c'est-à-dire qu'elle mobilise autant des procédures institutionnalisées que des processus informels. Ceux-ci font appel à la réflexivité de différents acteurs qui fabriquent les conditions de l'adaptation transformatrice (Berdoulay et Soubeyran, 2012). Dans cette perspective, il faut souligner que si l'adaptation est fondée sur le lieu, elle n'est pas pour autant à enfermer dans des territoires locaux. La multidimensionnalité des risques comme des liens sociaux, économiques, politiques et affectifs, fait que l'adaptation et la concertation doivent aussi être multiscalaires.

2 C'est-à-dire un problème vicieux, tordu, non réductible à une approche technique classique.
$\mathrm{Au}$ fond, l'approche de l'adaptation devient porteuse $d$ 'innovation dès lors qu'elle ne se replie pas sur un des deux termes d'une opposition à laquelle elle est exposée : la planification par le haut, et l'initiative locale (Tubiana et al., 2010). Or, chaque phase du processus (anticipation, réponse, reconstruction) peut être améliorée en jouant non plus sur un couple antagonique adaptation/capacité adaptative, mais au contraire en faisant en sorte que l'un soit condition d'amélioration de l'autre.

Ainsi, l'adaptation transformatrice se définit par le couplage de deux notions, et ce, à toute échelle. Il y a $d^{\prime}$ 'une part l'adaptation, vecteur d'une politique visant à fabriquer des comportements attendus, et d'autre part la capacité adaptative des populations locales. C'est pourquoi ce couplage encourage l'apprentissage et la concertation à tous les niveaux de l'action.

\section{Conclusion}

L'adaptation au changement climatique constitue pour la pensée planificatrice un enjeu qu'il n'est pas pertinent de réduire à un problème particulier qu'elle peut aborder avec ses approches classiques. Au contraire, comme nous l'avons vu, il y a tout intérêt à mettre en relation cet enjeu avec celui de la durabilité, comme souligné par les travaux faits sous le couvert d'une science de la durabilité. Nous n'avons pas ici argumenté en faveur des formulations particulières qu'elle a pu prendre institutionnellement. Nous en avons plutôt retenu certaines idées directrices qui ont l'avantage de proposer un regard nouveau sur l'adaptation en aménagement. Sa portée provient avant tout de la mise en cohérence des divers aspects de la question; à ce titre, la science de la durabilité présente l'intérêt majeur de dépasser la simple interdisciplinarité, au profit de la transversalité des questionnements et des démarches.

La façon dont l'adaptation a été introduite en aménagement en France, ainsi que d'autres préoccupations telles que la résilience, illustre l'intérêt de ce point de vue. En effet, ce qui caractérise l'introduction de l'adaptation, nous l'avons mentionné, c'est sa soudaineté. Les chercheurs ont été pris de court. La grande majorité des études et des recherches qui aujourd'hui traite de cette question s'inspire de ce qui a été produit à ce propos dans les pays de langue anglaise. Mais il est symptomatique que cela se fasse sans s'intéresser au cadrage qui y est discuté ou proposé. Or, c'est précisément le rôle que peut jouer la science de la durabilité.

Dans le champ de la planification environnementale, en France en particulier, la science de la durabilité a eu très peu d'échos jusqu'à présent. Cela peut étonner parce que, depuis le milieu des années 1990, les acteurs sociaux subissent, tous azimuts, des injonctions au 
développement durable ainsi qu'un appel à la mutation d'une pensée de l'action qui puisse aider à cette transition. Peut-être qu'en France - comme ailleurs - l'institutionnalisation des disciplines scientifiques a freiné l'ouverture transdisciplinaire que réclame la science de la durabilité. Mais peut-être aussi que l'ardente obligation de penser depuis quelques années l'aménagement non plus seulement à l'aune du développement durable, mais plus concrètement en matière $\mathrm{d}^{\prime}$ adaptation des territoires face au changement climatique, conduit à susciter un intérêt pour le cadrage qu'apporte la science de la durabilité.

Ce cadrage nous semble d'autant plus intéressant qu'il se construit sur un ensemble de tensions aptes à nourrir les réflexions sur l'adaptation. Ces tensions, articulées les unes aux autres, se déploient de multiples façons. Par exemple, la science de la durabilité se concentre sur l'étude des relations entre systèmes sociaux et systèmes naturels, sans fondre les uns avec les autres. La tension vient du fait que, s'il y a bien transformation réciproque entre la nature et la société (c'est l'enjeu que désigne de façon croissante le terme d'anthropocène), la nature fonctionne bel et bien comme entité relativement autonome, tout comme la société.

La tension se manifeste également entre un éthos scientifique et une position très située de cette activité. La science de la durabilité, rappelons-le, ne se veut ni une science fondamentale ni une science appliquée. Elle correspond à une volonté de faire science en même temps que d'être une aide à l'action : elle se veut utile, guidée par des problèmes pratiques à résoudre, alors même qu'elle se confronte à l'incertitude, aux processus non linéaires, à la complexité. C'est un champ scientifique qui se construit au croisement des compétences, des échelles multiples, des problèmes tels qu'ils se posent différemment selon le niveau de développement socioéconomique, ou encore les lieux et les territoires.

Replacée dans ce champ en tension, l'adaptation trouve les conditions d'une dynamique heuristique positive. Du point de vue de la planification, le sens qu'elle prend dans ce champ s'inscrit moins dans l'incrémentalisme que dans la rupture. C'est ce que nous avons cherché à définir en utilisant la notion d'adaptation transformatrice. La tension s'exerce entre l'injonction de l'adaptation et le renforcement de la capacité adaptative, instaurant l'apprentissage et la concertation comme les ressorts fondamentaux d'une action transformatrice.

Ainsi, le champ de préoccupations que dessine la science de la durabilité constitue un milieu, un cadre réflexif favorable à l'adaptation comme question d'aménagement. Mais il faut voir aussi que l'intérêt de cette science va plus loin, dépassant son apport à la seule question de l'adaptation. En effet, cette science permet de resituer les enjeux au sein de la société. De fait, le cadrage qu'elle apporte joue le rôle d'une interface avec les autres enjeux de la société. Pour reprendre une expression de Imre Lakatos, le champ de préoccupations structuré par la science de la durabilité peut servir de ceinture protectrice de la planification (comme en matière d'adaptation) vis-à-vis de la demande sociale (comme la commande publique), et ce, afin de faire avancer la transition vers le développement durable.

\section{Références}

Alexander, E.R., 1984. After rationality, what? A review of response to paradigm breakdown, Journal of the American Planning Association, 50, 1, 62-69.

Banque mondiale, 2011. Guide pour l'adaptation au changement climatique des villes. Résumé analytique.

Beck, U., 2001 [1986, éd. orig. allemande]. La Société du risque : sur la voie d'une autre modernité, Paris, Aubier.

Berdoulay, V., Soubeyran, O., 1996. Développement durable et débat public: expériences nord-américaines, Paris, Édition conjointe des ministères de l'Équipement et de l'Environnement, Coll. Villes et Territoires.

Berdoulay, V., Soubeyran, O. (Eds), 2000. Milieu, colonisation et développement durable : perspectives géographiques sur l'aménagement, Paris, L'Harmattan.

Berdoulay, V., Soubeyran, O., 2012. Pratiques réflexives en aménagement pour une adaptation aux changements environnementaux, L'Espace Géographique, 41, 2, 169-180.

Burton, I., Kates, R.W., White, G.F., 1978. The Environment as Hazard, New York, Oxford University Press.

Burton, I., Huq, S., Lim, B., Pilifosova, O., Schipper, E.L., 2002. From impacts assessment to adaptation priorities: The shaping of adaptation policy, Climate Policy, 2, 145-159.

Chalas, Y., Soubeyran, O., 2010. Incertitude, environnement et aménagement, quelle rupture ?, in Chalas, Y., Gilbert, C., Vinck, D. (Eds), Comment les acteurs s'arrangent avec l'incertitude, Paris, Éditions des archives contemporaines, 135-157.

Clark, W.C., 2007. Sustainability science: A room of its own, Proceedings of the National Academy of Sciences (USA), 104, 6, 1737-1738.

Clark, W.C., Dickson, N.M., 2003. Sustainability science: The emerging research program, Proceedings of the National Academy of Science (USA), 100, 8059-8061.

Clark, W.C., Munn, R.E. (Eds), 1986. Sustainable Development of the Biosphere, Cambridge, Cambridge University Press.

Clark, W.C., Crutzen, P.J., Schellnhuber, H.J., 2004. Science for global sustainability: Toward a new paradigm, in Schellnhuber, H.J., Crutzen, P.J., Clark, W.C., Claussen, M., Held, H. (Eds), Earth Systems Analysis for Sustainability, Cambridge (MA), MIT Press, 1-28.

Claval, P., 2001. Épistémologie de la géographie, Paris, Nathan.

CMED (Commission mondiale pour l'environnement et le développement), 1987. Our Common Future, Oxford, Oxford University Press [trad.: Notre avenir à tous, Montréal, Éditions du Fleuve, 1988]. 
Colten, C.E., Kates, R.W., Laska, S.B., 2008. Three years after Katrina: Lessons for community resilience, Environment: Science and Policy for Sustainable Development, 50, 5, 36-47.

Elster, J., 1987. Le Laboureur et ses enfants: deux essais sur les limites de la rationalité, Paris, Éditions de Minuit.

Entrikin, J.N., 2007. Place destruction and cultural trauma, in Alexander, J.C., Reed, I. (Eds.), in Culture, Society, and Democracy: The Interpretive Approach, Boulder (CO), Paradigm Press, 163-179.

Fabiani, J.-L., Theys, J. (Eds), 1987. La Société vulnérable : évaluer et maîtriser les risques, Paris, Presses de l'École normale supérieure.

Folke, C., Carpenter, S., Elmqvist, T., Gunderson, L., Holling, C.S., Walker, B., 2002a. Resilience and sustainable development: Building adaptive capacity in a world of transformations, Ambio, 31, 5, 437-440.

Folke, C., Carpenter, S., Elmqvist, T., Gunderson, L., Holling, C.S., Walker, B., Bengtsson, J., Berkes, F., Colding, J., Danell, K., Falkenmark, M., Gordon, L., Kasperson, R., Kautsky, N., Kinzig, A., Levin, S., Mäler, K.-G., Moberg, F., Ohlsson, L., Olsson, P., Ostrom, E., Reid, W., Rockström, J., Savenije, H., Svedin, U., 2002b. Resilience and Sustainable Development: Building Adaptive Capacity in a World of Transformation, Stockholm, Ministère de l'Environnement (Swedish environmental advisory council).

Folke, C., Hahn, T., Olsson, P., Norberg, J., 2005. Adaptive governance of social-ecological systems, Annual Review of Environment and Resources, 30, 441-473.

Füssel, H.-M., 2007. Vulnerability: A generally applicable conceptual framework for climate change research, Global Environmental Change, 17, 155-167.

Giddens, A., 2011. The Politics of Climate Change, Cambridge, Polity.

Glacken, C.J., 2000-2007. Histoire de la pensée géographique, Paris, CTHS.

Gross, M., 2010. Ignorance and Surprise: Science, Society, and Ecological Design, Cambridge (MA), MIT Press.

Holling, C.S. (Ed.), 1978. Adaptive Environmental Assessment and Management, Chichester (NY), John Wiley \& Sons.

Hulme, M., 2009. Why We Disagree about Climate Change: Understanding Controversy, Inaction and Opportunity, Cambridge, Cambridge University Press.

Jollivet, M. (Ed.), 2001. Le Développement durable, de l'utopie au concept: de nouveaux chantiers pour la recherche, Paris, Elsevier.

Kates, R.W., 1974. Comprehensive environmental planning, in Regional Planning: Challenge and Prospects, New York, Frederick A. Praeger, 67-87.

Kates, R.W., 2000. Navigating the future: A transition toward sustainability, in Lo, F., Tokuda, H., Cooray, N.S. (Eds), The Sustainable Future of the Global System, III. Proceedings of International Conference on Sustainable Future of Global System, 24-25 May, Tokyo, United Nations University, 25-35.

Kates, R.W., Clark, W.C., Corell, R., Hall, J.M., Jaeger, C.C., Lowe, I., McCarthy, J.J., Schellnhuber, H.J., Bolin, B., Dickson, N.M., Faucheux, S., Gallopin, G.C., Grubler, A., Huntley, B., Jager, J., Jodha, N.S., Kasperson, R.E., Mabogunje, A., Matson, P., Mooney, H., Moore, B., O'Riordan, T., Svedin, U., 2001. Sustainability science, Science, 292, 641-642.
Kates, R.W., Travis, W.R., Wilbanks, T.J., 2012. Transformational adaptation when incremental adaptations to climate change are insufficient, Proceedings of the National Academy of Science (USA), 109, 7156-7161.

Kay, R., 2010. Anticipatory governance: A tool for climate change adaptation, Journal of the American Planning Association, 76, 4, 496-511.

Komiyama, H., Takeuchi, K., 2006. Sustainability science, Sustainability Science, 1, 1-6.

Levin, K., Cashore, B., Bernstein, S., Auld, G., 2010. Playing it forward: Path dependency, progressive incrementalism, and the "supper wicked" problem of global climate change, (online: http:// environment.research.yale.edu/documents/downloads/09/2010_super_wicked_levin_cashore_bernstein_auld.pdf).

Lavoux, T., Theys, J. (Eds), 2001. Propositions d'indicateurs de développement durable pour la France, Paris, Ifen (Institut français de l'environnement), Études et Travaux $\mathrm{n}^{\mathrm{O}} 35$.

Leiserowitz, A.A., Kates, R.W., Parris, T.M., 2006. Sustainability values, attitudes, and behaviors: A review of multinational and local trends, Annual Review of Environment and Resources, 31, 413-444.

Lindblom, C.E., 1959. The science of muddling through, Public Administration Review, 19, 2, 79-88.

Lindblom, C.E., 1979. Still muddling, not yet through, Public Administration Review, 39, 6, 517-526.

Liu, J., Dietz, T., Cerpenter, R.S., Alberti, M., Folke, C., Moran, E., Pell, A.N., Deadman, P., Kratz, T., Lubchenko, J., Ostrom, E., Ouyang, Z., Provencher, W., Redman, C.L., Schneider, S.H., Taylor, W.W., 2007. Complexity of coupled human and natural systems, Science, 317, 1513-1516.

Lynam, T., Wil de Jong, D.S., Sheil, D., Kusumanto, T., Evans, K., 2007. A review of tools for incorporating community knowledge, preferences, and values into decision making in natural resources management, Ecology and Society, 12, 1 (online: http://www.ecologyandsociety.org/vol12/iss1/ $\operatorname{art5} /)$.

Magnan, A., 2010. Questions de recherche autour de l'adaptation au changement climatique, Natures Sciences Sociétés, 18, 329-333.

NRC (National Research Council) - Board on sustainable development, 1999. Our Common Journey: A transition Toward Sustainability, Washington D.C., National Academy Press.

O’Brien, K., Eriksen, S., Schjolden, A., Nygaard, L., 2004. What's in a word ? Conflicting interpretations of vulnerability in climate change research, Oslo, CICERO (Center for international climate and environmental research), Working Paper 4 (online: http://www.cicero.uio.no/ media/2682.pdf).

Onerc (Observatoire national sur les effets du réchauffement climatique), 2005. Un climat à la dérive : comment s'adapter. Rapport au Premier ministre et au Parlement, Paris, La Documentation française.

Onerc, 2007. Stratégie nationale d'adaptation au changement climatique. Rapport au Premier ministre et au Parlement, Paris, La Documentation française.

Onerc, 2010. Villes et adaptation au changement climatique. Rapport au Premier ministre et au Parlement, Paris, La Documentation française. 
Onerc, 2012. L'Adaptation de la France au changement climatique : rapport au Premier ministre et au Parlement, Paris, La Documentation française.

Perthuis, C. (de), Hallegate, S., Lecocq, F., 2010. Économie de l'adaptation au changement climatique. Rapport au Conseil économique pour le développement durable, Ministère de l'Écologie, de l'Énergie, du Développement durable et de la Mer, Paris.

Sachs, I., 1980. Stratégies de l'écodévelopement, Paris, Éditions Ouvrières.

Soubeyran, O., 1988. Malaise dans la planification, Annales de la Recherche Urbaine, 37, 24-30.

Soubeyran, O., 2000. Définir les politiques : l'étude d'impact de l'aménagement en environnement, in Renard, D., Caillosse, J., Béchillon, D. (de) [Eds], Droit et Société, Série Politique, 30, Paris, L.G.D.J., 183-206.

Stokes, D.E., 1997. Pasteur's Quadrant : Basic Science and Technological Innovation, Washington D.C., Brookings Institution Press.
Theys, J., du Tertre, C., Rauschmayer, F., 2010. Le Développement durable, la seconde étape, La Tour-d'Aigues, Éditions de l'Aube.

Tubiana, L., Gemenne, F., Magnan, A., 2010. Anticiper pour s'adapter : le nouvel enjeu du changement climatique, Paris, Pearson Éducation.

Turner II, B.L., Kasperson, R.E., Matson, P., MacCathy, J.J., Corell, R.W., Christensen, L., Eckley, N., Kasperson, J.X., Luers, A., Martello, M.L., Mathisen, C.S., Polsky, C., Pulsipher, A., Schiller, A., Tyler, N., 2003. Framework for vulnerability analysis in sustainability science, Proceedings of the National Academy of Science (USA), 100, 14, 8074-8079.

Wilbanks, T.J., Kates, R.W., 2010. Beyond adapting to climate change: Embedding adaptation in responses to multiple threats and stresses, Annals of the Association of American Geographers, 100, 4, 719-728.

Zaccaï, E., 2002. Le Développement durable, dynamique et constitution d'un projet, Bruxelles, PIE Peter Lang.

Zaccaï, E., Gemene, F., Decroly, J.M., 2012. Controverses climatiques, sciences et politique, Paris, Presses de Sciences Po.

Reçu le 19 mars 2012. Accepté le 7 novembre 2013. 